\title{
Spondylometaphyseal dysplasia-bowed forearms-facial dysmorphism syndrome
}

INSERM

\section{Source}

INSERM. (1999). Orphanet: an online rare disease and orphan drug data base.

Spondylometaphyseal dysplasia-bowed forearms-facial dysmorphism syndrome.

ORPHA:168552

Spondylometaphyseal dysplasia-bowed forearms-facial dysmorphism syndrome is a rare, genetic, primary bone dysplasia disorder characterized by short stature,

hyperlordosis, protuberant abdomen, mild bilateral genu varum, bowed and shortened forearms with limited elbow extension, and discrete facial dysmorphism (prominent forehead, hypertelorism, flat nasal bridge). Radiographically, moderate platyspondyly, including posterior wedging with anterior bullet-shaped vertebral bodies, with minimal metaphyseal abnormalities are observed. 